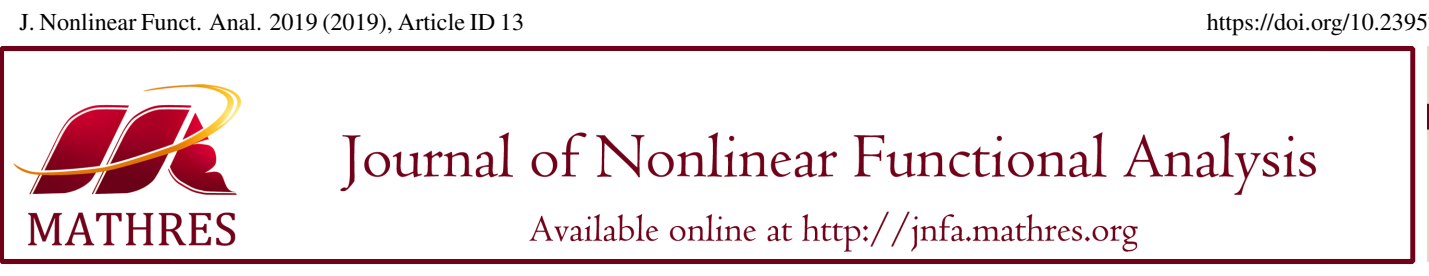

https://doi.org/10.23952/jnfa.2019.13

\title{
SOME NEW RESULTS ON COMMON COUPLED FIXED POINTS OF TWO HYBRID PAIRS OF MAPPINGS IN PARTIAL METRIC SPACES
}

\author{
FENG GU $^{1, *}$, WASFI SHATANAWI ${ }^{2,3}$ \\ ${ }^{1}$ Department of Mathematics, Hangzhou Normal University, Hangzhou, Zhejiang 310036, China \\ ${ }^{2}$ Department of Mathematics and General Courses, Prince Sultan University, Riyadh, Saudi Arabia \\ ${ }^{3}$ Department of Medical Research, China Medical University Hospital, China Medical University, Taichung 40402, Taiwan
}

\begin{abstract}
By using the concept of $w$-compatible mappings, we establish some new common coupled fixed point theorems for two hybrid pairs of mappings satisfying a symmetric type contractive condition in a partial metric space. We do not use the continuity of any mapping for finding the coupled coincidence and common coupled fixed points. We also provide some examples to support our new results.
\end{abstract}

Keywords. Common coupled fixed point; Coupled coincidence point; Compatible mapping pair; Partial metric space.

2010 Mathematics Subject Classification. 47H10, 54H25.

\section{INTRODUCTION}

The concept of partial metric spaces was introduced by Matthews [1] in 1994. A partial metric space is a generalized metric space. In a partial metric space, the distance of a point to its self may not be zero. In [1], Matthews extended the Banach contraction principle from metric spaces to partial metric spaces. Based on the concept of partial metric spaces, several authors obtained some fixed point results for mappings satisfying different contractive conditions; see, e.g., [2, 3, 4, 5, 6, 7, 8, 9] and the references therein. Recently, Haghi, Rezapour and Shahzad [10] proved that some of fixed point theorems in partial metric spaces can be obtained from metric spaces.

The definition of partial metric spaces is given as follows.

Definition 1.1. [1] A partial metric on a nonempty set $X$ is a function $p: X \times X \longrightarrow \mathbb{R}^{+}$such that, for all $x, y, z \in X$,

$$
\begin{aligned}
& \left(p_{1}\right) x=y \Leftrightarrow p(x, x)=p(x, y)=p(y, y) \\
& \left(p_{2}\right) p(x, . x) \leq p(x, y) \\
& \left(p_{3}\right) p(x, y)=p(y, x)
\end{aligned}
$$

\footnotetext{
${ }^{*}$ Corresponding author.
}

E-mail addresses: mathgufeng@163.com (F. Gu), wshatanawi@psu.edu.sa (W. Shatanawi).

Received September 16, 2018; Accepted February 25, 2019. 
$\left(p_{4}\right) p(x, y) \leq p(x, z)+p(z, y)-p(z, z)$.

A partial metric space is a pair $(X, p)$ such that $X$ is a nonempty set and $p$ is a partial metric on $X$.

Remark 1.2. It is clear that if $p(x, y)=0$, then one has from $\left(p_{1}\right)$ and $\left(p_{2}\right)$ that $x=y$. But if $x=y$, then $p(x, y)$ may not be 0 .

Each partial metric $p$ on $X$ generates a $T_{0}$ topology $\tau_{p}$ on $X$, which has as base the family of open $p$-balls $\left\{B_{p}(x, \varepsilon): x \in X, \varepsilon>0\right\}$, where $B_{p}(x, \varepsilon)=\{y \in X: p(x, y)<p(x, x)+\varepsilon\}$ for all $x \in X$ and $\varepsilon>0$.

It is remarkable that if $p$ is a partial metric on $X$, then the function $d_{p}: X \times X \rightarrow \mathbb{R}^{+}$given by

$$
d_{p}(x, y)=2 p(x, y)-p(x, x)-p(y, y)
$$

is a usual metric on $X$.

Definition 1.3. [1,7] Let $(X, p)$ be a partial metric space. Then

(i) a sequence $\left\{x_{n}\right\}$ in a partial metric space $(X, p)$ is said to be convergent to a point $x \in X$ if and only if $p(x, x)=\lim _{n \rightarrow \infty} p\left(x, x_{n}\right)$;

(ii) a sequence $\left\{x_{n}\right\}$ in a partial metric space $(X, p)$ is called a Cauchy sequence if there exists (and is finite) $\lim _{n, m \rightarrow \infty} p\left(x_{n}, x_{m}\right)$;

(iii) a partial metric space $(X, p)$ is said to be complete if every Cauchy sequence $\left\{x_{n}\right\}$ in $X$ converges, with respect to $\tau_{p}$, to a point $x \in X$ such that $p(x, x)=\lim _{n, m \rightarrow \infty} p\left(x_{n}, x_{m}\right)$.

In 2006, Bhaskar and Lakshmikantham [11] introduced the concept of coupled fixed points and studied some nice coupled fixed point theorems. Later, Lakshmikantham and Ćirić [12] introduced the definition of a coupled coincidence point of mappings. For recent results on a coupled fixed point, we refer the reader to $[13,14,15,16,17]$.

Definition 1.4. [11] Let $X$ be a nonempty set. We call an element $(x, y) \in X \times X$ a coupled fixed point of the mapping $F: X \times X \rightarrow X$ if $F(x, y)=x$ and $F(y, x)=y$.

Definition 1.5. [12] An element $(x, y) \in X \times X$ is called

(i) a coupled coincidence point of the mapping $F: X \times X \rightarrow X$ and $g: X \rightarrow X$ if $F(x, y)=g x$ and $F(y, x)=g y$

(ii) a common coupled fixed point of mappings $F: X \times X \rightarrow X$ and $g: X \rightarrow X$ if $F(x, y)=g x=x$ and $F(y, x)=g y=y$.

In 2010, Abbas, Khan and Radenovic [18] introduced the concept of $w$-compatible mappings as follows.

Definition 1.6. Let $X$ be a nonempty set. We say that the mappings $F: X \times X \rightarrow X$ and $g: X \rightarrow X$ are $w$-compatible if $g F(x, y)=F(g x, g y)$ whenever $g x=F(x, y)$ and $g y=F(y, x)$.

Recently, Aydi [13] obtained the following.

Theorem 1.7. Let $(X, p)$ be a partial metric space. Suppose that the mapping $F: X \times X \rightarrow X$ satisfies the following contractive condition, for all $x, y, u, v \in X$,

$$
p(F(x, y), F(u, v)) \leq k p(x, u)+l p(y, v),
$$

where $k, l$ are nonnegative constants with $k+l<1$. Then $F$ has a unique coupled fixed point. 
The purpose of this paper is to use the concept of $w$-compatible mappings to discuss some new common coupled fixed point problems for two hybrid pairs of mappings in the framework of partial metric spaces. We do not use the continuity of any mapping for finding the coupled coincidence and common coupled fixed points. The results presented in this paper extend and improve some known corresponding results in the literature.

We need the following useful lemmas to prove our main results.

Lemma 1.8. [2] Let $(X, p)$ be a partial metric space. Then the following statements hold

(1) if $p(x, y)=0$, then $x=y$;

(2) if $x \neq y$, then $p(x, y)>0$.

Lemma 1.9. $[1,4,7]$ Let $(X, p)$ be a partial metric space.

(1) $\left\{x_{n}\right\}$ is a Cauchy sequence in $(X, p)$ if and only if it is a Cauchy sequence in the metric space $\left(X, d_{p}\right)$.

(2) A partial metric space $(X, p)$ is complete if and only if the metric space $\left(X, d_{p}\right)$ is complete. Moreover,

$$
\lim _{n \rightarrow \infty} d_{p}\left(x, x_{n}\right)=0 \Leftrightarrow \lim _{n \rightarrow \infty} p\left(x, x_{n}\right)=\lim _{n, m \rightarrow \infty} p\left(x_{n}, x_{m}\right)=p(x, x) .
$$

Lemma 1.10. $[2,3,8]$ Let $(X, p)$ be a partial metric space and $x_{n} \rightarrow z$ with $p(z, z)=0$. Then $\lim _{n \rightarrow \infty} p\left(x_{n}, y\right)=$ $p(z, y)$ for every $y \in X$.

\section{MAIN RESULTS}

Theorem 2.1. Let $(X, p)$ be a partial metric space. Let $F, G: X \times X \rightarrow X, f, g: X \rightarrow X$ be four mappings. Suppose that there exist $k_{1}, k_{2}, k_{3}, k_{4}$ and $k_{5}$ in $[0,1)$ with

$$
k_{1}+k_{2}+k_{3}+2 k_{4}+2 k_{5}<1
$$

such that

$$
\begin{aligned}
& p(F(x, y), G(u, v))+p(F(y, x), G(v, u)) \\
& \leq \quad k_{1}[p(f x, g u)+p(f y, g v)]+k_{2}[p(f x, F(x, y))+p(f y, F(y, x))] \\
&+k_{3}[p(g u, G(u, v))+p(g v, G(v, u))]+k_{4}[p(f x, G(u, v))+p(f y, G(v, u))] \\
&+k_{5}[p(g u, F(x, y))+p(g v, F(y, x))]
\end{aligned}
$$

for all $x, y, u, v \in X$. Also, suppose the following hypotheses:

(i) $F(X \times X) \subset g(X)$ and $G(X \times X) \subset f(X)$,

(ii) either $f(X)$ or $g(X)$ is a complete subspace of $X$,

(iii) $(F, f)$ and $(G, g)$ are w-compatible.

Then $F, G, f$ and $g$ have a unique common coupled fixed point in $X \times X$. Moreover, the common coupled fixed point of $F, G, f$ and $g$ has the form $(u, u)$.

Proof. Let $x_{0}, y_{0} \in X$. From (i), there exist sequences $\left\{x_{n}\right\},\left\{y_{n}\right\},\left\{z_{n}\right\}$ and $\left\{w_{n}\right\}$ in $X$ such that

$$
\left\{\begin{array}{l}
F\left(x_{2 n}, y_{2 n}\right)=g x_{2 n+1}=z_{2 n}, \forall n \geq 0, \\
F\left(y_{2 n}, x_{2 n}\right)=g y_{2 n+1}=w_{2 n}, \forall n \geq 0, \\
G\left(x_{2 n+1}, y_{2 n+1}\right)=f x_{2 n+2}=z_{2 n+1}, \forall n \geq 0, \\
G\left(y_{2 n+1}, x_{2 n+1}\right)=f y_{2 n+2}=w_{2 n+1}, \forall n \geq 0 .
\end{array}\right.
$$


It follows from (2.2), $\left(p_{2}\right)$ and $\left(p_{4}\right)$ that

$$
\begin{aligned}
p\left(z_{2 n},\right. & \left.z_{2 n+1}\right)+p\left(w_{2 n}, w_{2 n+1}\right)=p\left(F\left(x_{2 n}, y_{2 n}\right), G\left(x_{2 n+1}, y_{2 n+1}\right)\right)+p\left(F\left(y_{2 n}, x_{2 n}\right), G\left(y_{2 n+1}, x_{2 n+1}\right)\right) \\
\leq \quad & k_{1}\left[p\left(f x_{2 n}, g x_{2 n+1}\right)+p\left(f y_{2 n}, g y_{2 n+1}\right)\right]+k_{2}\left[p\left(f x_{2 n}, F\left(x_{2 n}, y_{2 n}\right)\right)+p\left(f y_{2 n}, F\left(y_{2 n}, x_{2 n}\right)\right)\right] \\
& +k_{3}\left[p\left(g x_{2 n+1}, G\left(x_{2 n+1}, y_{2 n+1}\right)\right)+p\left(g y_{2 n+1}, G\left(y_{2 n+1}, x_{2 n+1}\right)\right)\right] \\
& +k_{4}\left[p\left(f x_{2 n}, G\left(x_{2 n+1}, y_{2 n+1}\right)\right)+p\left(f y_{2 n}, G\left(y_{2 n+1}, x_{2 n+1}\right)\right)\right] \\
& +k_{5}\left[p\left(g x_{2 n+1}, F\left(x_{2 n}, y_{2 n}\right)\right)+p\left(g y_{2 n+1}, F\left(y_{2 n}, x_{2 n}\right)\right)\right] \\
= & k_{1}\left[p\left(z_{2 n-1}, z_{2 n}\right)+p\left(w_{2 n-1}, w_{2 n}\right)\right]+k_{2}\left[p\left(z_{2 n-1}, z_{2 n}\right)+p\left(w_{2 n-1}, w_{2 n}\right)\right] \\
& +k_{3}\left[p\left(z_{2 n}, z_{2 n+1}\right)+p\left(w_{2 n}, w_{2 n+1}\right)\right]+k_{4}\left[p\left(z_{2 n-1}, z_{2 n+1}\right)+p\left(w_{2 n-1}, w_{2 n+1}\right)\right] \\
& +k_{5}\left[p\left(z_{2 n}, z_{2 n}\right)+p\left(w_{2 n}, w_{2 n}\right)\right] \\
\leq \quad & \left(k_{1}+k_{2}\right)\left[p\left(z_{2 n-1}, z_{2 n}\right)+p\left(w_{2 n-1}, w_{2 n}\right)\right]+k_{3}\left[p\left(z_{2 n}, z_{2 n+1}\right)+p\left(w_{2 n}, w_{2 n+1}\right)\right] \\
& +k_{4}\left[p\left(z_{2 n-1}, z_{2 n}\right)+p\left(z_{2 n}, z_{2 n+1}\right)-p\left(z_{2 n}, z_{2 n}\right)+p\left(w_{2 n-1}, w_{2 n}\right)+p\left(w_{2 n}, w_{2 n+1}\right)\right. \\
& \left.-p\left(w_{2 n}, w_{2 n}\right)\right]+k_{5}\left[p\left(z_{2 n}, z_{2 n+1}\right)+p\left(w_{2 n}, w_{2 n+1}\right)\right] \\
\leq \quad & \left(k_{1}+k_{2}+k_{4}\right)\left[p\left(z_{2 n-1}, z_{2 n}\right)+p\left(w_{2 n-1}, w_{2 n}\right)\right] \\
& +\left(k_{3}+k_{4}+k_{5}\right)\left[p\left(z_{2 n}, z_{2 n+1}\right)+p\left(w_{2 n}, w_{2 n+1}\right)\right]
\end{aligned}
$$

which implies that

$$
p\left(z_{2 n}, z_{2 n+1}\right)+p\left(w_{2 n}, w_{2 n+1}\right) \leq \frac{k_{1}+k_{2}+k_{4}}{1-k_{3}-k_{4}-k_{5}}\left[p\left(z_{2 n-1}, z_{2 n}\right)+p\left(w_{2 n-1}, w_{2 n}\right)\right] .
$$

Similarly, we can obtain

$$
\begin{aligned}
& p\left(z_{2 n-1}, z_{2 n}\right)+p\left(w_{2 n-1}, w_{2 n}\right)=p\left(F\left(x_{2 n}, y_{2 n}\right), G\left(x_{2 n-1}, y_{2 n-1}\right)\right)+p\left(F\left(y_{2 n}, x_{2 n}\right), G\left(y_{2 n-1}, x_{2 n-1}\right)\right) \\
& \leq \quad k_{1}\left[p\left(f x_{2 n}, g x_{2 n-1}\right)+p\left(f y_{2 n}, g y_{2 n-1}\right)\right]+k_{2}\left[p\left(f x_{2 n}, F\left(x_{2 n}, y_{2 n}\right)\right)+p\left(f y_{2 n}, F\left(y_{2 n}, x_{2 n}\right)\right)\right] \\
&+ k_{3}\left[p\left(g x_{2 n-1}, G\left(x_{2 n-1}, y_{2 n-1}\right)\right)+p\left(g y_{2 n-1}, G\left(y_{2 n-1}, x_{2 n-1}\right)\right)\right] \\
&+k_{4}\left[p\left(f x_{2 n}, G\left(x_{2 n-1}, y_{2 n-1}\right)\right)+p\left(f y_{2 n}, G\left(y_{2 n-1}, x_{2 n-1}\right)\right)\right] \\
&+k_{5}\left[p\left(g x_{2 n-1}, F\left(x_{2 n}, y_{2 n}\right)\right)+p\left(g y_{2 n-1}, F\left(y_{2 n}, x_{2 n}\right)\right)\right] \\
&= k_{1}\left[p\left(z_{2 n-1}, z_{2 n-2}\right)+p\left(w_{2 n-1}, w_{2 n-2}\right)\right]+k_{2}\left[p\left(z_{2 n-1}, z_{2 n}\right)+p\left(w_{2 n-1}, w_{2 n}\right)\right] \\
&+k_{3}\left[p\left(z_{2 n-2}, z_{2 n-1}\right)+p\left(w_{2 n-2}, w_{2 n-1}\right)\right]+k_{4}\left[p\left(z_{2 n-1}, z_{2 n-1}\right)+p\left(w_{2 n-1}, w_{2 n-1}\right)\right] \\
&+k_{5}\left[p\left(z_{2 n-2}, z_{2 n}\right)+p\left(w_{2 n-2}, w_{2 n}\right)\right] \\
& \leq \quad\left(k_{1}+k_{3}\right)\left[p\left(z_{2 n-2}, z_{2 n-1}\right)+p\left(w_{2 n-2}, w_{2 n-1}\right)\right]+k_{2}\left[p\left(z_{2 n-1}, z_{2 n}\right)+p\left(w_{2 n-1}, w_{2 n}\right)\right] \\
&+k_{4}\left[p\left(z_{2 n-1}, z_{2 n}\right)+p\left(w_{2 n-1}, w_{2 n}\right)\right] \\
&+k_{5}\left[p\left(z_{2 n-2}, z_{2 n-1}\right)+p\left(z_{2 n-1}, z_{2 n}\right)-p\left(z_{2 n-1}, z_{2 n-1}\right)+p\left(w_{2 n-2}, w_{2 n-1}\right)+p\left(w_{2 n-1}, w_{2 n}\right)\right. \\
&\left.-p\left(w_{2 n-1}, w_{2 n-1}\right)\right] \\
& \leq \quad\left(k_{1}+k_{3}+k_{5}\right)\left[p\left(z_{2 n-2}, z_{2 n-1}\right)+p\left(w_{2 n-2}, w_{2 n-1}\right)\right] \\
&+\left(k_{2}+k_{4}+k_{5}\right)\left[p\left(z_{2 n-1}, z_{2 n}\right)+p\left(w_{2 n-1}, w_{2 n}\right)\right]
\end{aligned}
$$


which implies that

$$
p\left(z_{2 n-1}, z_{2 n}\right)+p\left(w_{2 n-1}, w_{2 n}\right) \leq \frac{k_{1}+k_{3}+k_{5}}{1-k_{2}-k_{4}-k_{5}}\left[p\left(z_{2 n-2}, z_{2 n-1}\right)+p\left(w_{2 n-2}, w_{2 n-1}\right)\right] .
$$

By combining (2.4) and (2.5), we obtain

$$
p\left(z_{n}, z_{n+1}\right)+p\left(w_{n}, w_{n+1}\right) \leq k\left[p\left(z_{n-1}, z_{n}\right)+p\left(w_{n-1}, w_{n}\right)\right],
$$

where

$$
k=\max \left\{\frac{k_{1}+k_{2}+k_{4}}{1-k_{3}-k_{4}-k_{5}}, \frac{k_{1}+k_{3}+k_{5}}{1-k_{2}-k_{4}-k_{5}}\right\} .
$$

Obviously, $0 \leq k<1$. Repetition of the above inequality (2.6) $n$-times, we get

$$
p\left(z_{n}, z_{n+1}\right)+p\left(w_{n}, w_{n+1}\right) \leq k^{n}\left[q_{1}\left(z_{0}, z_{1}\right)+q_{1}\left(w_{0}, w_{1}\right)\right] .
$$

Next, we prove that $\left\{z_{n}\right\}$ and $\left\{w_{n}\right\}$ are Cauchy sequences in $X$.

In fact, for each $n, m \in \mathbb{N}, m>n$, we obtani from $\left(p_{4}\right)$ and (2.7) that

$$
\begin{aligned}
p\left(z_{n}, z_{m}\right)+p\left(w_{n}, w_{m}\right) & \leq \sum_{i=n}^{m-1}\left[p\left(z_{i}, z_{i+1}\right)+p\left(w_{i}, w_{i+1}\right)\right] \\
& \leq \sum_{i=n}^{m-1} k^{i}\left[p\left(z_{0}, z_{1}\right)+p\left(w_{0}, w_{1}\right)\right] \\
& \leq \frac{k^{n}}{1-k}\left[p\left(z_{0}, z_{1}\right)+p\left(w_{0}, w_{1}\right)\right] .
\end{aligned}
$$

This implies that

$$
\lim _{n, m \rightarrow \infty}\left[p\left(z_{n}, z_{m}\right)+p\left(w_{n}, w_{m}\right)\right]=0 .
$$

It follows that

$$
\lim _{n, m \rightarrow \infty} p\left(z_{n}, z_{m}\right)=0 \text { and } \lim _{n, m \rightarrow \infty} p\left(w_{n}, w_{m}\right)=0 .
$$

Hence $\left\{z_{n}\right\}$ and $\left\{w_{n}\right\}$ are Cauchy sequences in the partial metric space $(X, p)$. By Lemma 1.9, $\left\{z_{n}\right\}$ and $\left\{w_{n}\right\}$ are Cauchy sequences in $\left(X, d_{p}\right)$. Therefore, $\left\{z_{2 n+1}\right\}$ and $\left\{w_{2 n+1}\right\}$ are Cauchy sequences in the subspace $\left(f(X), d_{p}\right)$. Suppose that $f(X)$ is complete. Since $\left\{z_{2 n+1}\right\} \subset f(X)$ and $\left\{w_{2 n+1}\right\} \subset f(X)$ are Cauchy sequences in $\left(f(X), d_{p}\right)$, it follows that the sequences $\left\{z_{2 n+1}\right\}$ and $\left\{w_{2 n+1}\right\}$ are convergent in $\left(f(X), d_{p}\right)$. Hence, there exist $u, v \in f(X)$ such that

$$
\lim _{n \rightarrow \infty} d_{p}\left(z_{2 n+1}, u\right)=0 \text { and } \lim _{n \rightarrow \infty} d_{p}\left(w_{2 n+1}, v\right)=0 .
$$

Since $u, v \in f(X)$, there exist $s, t \in X$ such that $u=f s$ and $v=f t$. Since $\left\{z_{n}\right\}$ and $\left\{w_{n}\right\}$ are Cauchy sequences in $X$ and $\left\{z_{2 n+1}\right\} \rightarrow u$ and $\left\{w_{2 n+1}\right\} \rightarrow v$, it follows that $\left\{z_{2 n}\right\} \rightarrow u$ and $\left\{w_{2 n}\right\} \rightarrow v$. From Lemma 1.9, we have

$$
p(u, u)=\lim _{n \rightarrow \infty} p\left(z_{2 n}, u\right)=\lim _{n \rightarrow \infty} p\left(z_{2 n+1}, u\right)=\lim _{n, m \rightarrow \infty} p\left(z_{n}, z_{m}\right)
$$

and

$$
p(v, v)=\lim _{n \rightarrow \infty} p\left(w_{2 n}, v\right)=\lim _{n \rightarrow \infty} p\left(w_{2 n+1}, v\right)=\lim _{n, m \rightarrow \infty} p\left(w_{n}, w_{m}\right) .
$$

Combining (2.9), (2.10) with (2.11), we have that

$$
p(u, u)=0=p(v, v) .
$$


By $\left(p_{4}\right)$, we obtain

$$
\begin{aligned}
p\left(F(s, t), z_{2 n+1}\right) & \leq p(F(s, t), u)+p\left(u, z_{2 n+1}\right)-p(u, u) \\
& =p(F(s, t), u)+p\left(u, z_{2 n+1}\right) \\
& \leq p\left(F(s, t), z_{2 n+1}\right)+p\left(z_{2 n+1}, u\right)-p\left(z_{2 n+1}, z_{2 n+1}\right)+p\left(u, z_{2 n+1}\right) \\
& \leq p\left(F(s, t), z_{2 n+1}\right)+p\left(z_{2 n+1}, u\right)+p\left(u, z_{2 n+1}\right) .
\end{aligned}
$$

Letting $n \rightarrow \infty$ in the above inequalities and using (2.10) and (2.12), we have

$$
\lim _{n \rightarrow \infty} p\left(F(s, t), z_{2 n+1}\right) \leq p(F(s, t), u) \leq \lim _{n \rightarrow \infty} p\left(F(s, t), z_{2 n+1}\right) .
$$

That is,

$$
\lim _{n \rightarrow \infty} p\left(F(s, t), z_{2 n+1}\right)=p(F(s, t), u) .
$$

Similarly, using (2.11) and (2.12), we have

$$
\lim _{n \rightarrow \infty} p\left(F(t, s), w_{2 n+1}\right)=p(F(t, s), v) .
$$

Now we prove that $F(s, t)=u=f s$ and $F(t, s)=v=f t$. In fact, it follows from (2.2) and (2.3) that

$$
\begin{aligned}
& p\left(F(s, t), z_{2 n+1}\right)+p\left(F(t, s), w_{2 n+1}\right) \\
&=\quad p\left(F(s, t), G\left(x_{2 n+1}, y_{2 n+1}\right)\right)+p\left(F(t, s), G\left(y_{2 n+1}, x_{2 n+1}\right)\right) \\
& \leq \quad k_{1}\left[p\left(f s, g x_{2 n+1}\right)+p\left(f t, g y_{2 n+1}\right)\right]+k_{2}[p(f s, F(s, t))+p(f t, F(t, s))] \\
&+ k_{3}\left[p\left(g x_{2 n+1}, G\left(x_{2 n+1}, y_{2 n+1}\right)\right)+p\left(g y_{2 n+1}, G\left(y_{2 n+1}, x_{2 n+1}\right)\right)\right] \\
&+k_{4}\left[p\left(f s, G\left(x_{2 n+1}, y_{2 n+1}\right)\right)+p\left(f t, G\left(y_{2 n+1}, x_{2 n+1}\right)\right)\right] \\
&+k_{5}\left[p\left(g x_{2 n+1}, F(s, t)\right)+p\left(g y_{2 n+1}, F(t, s)\right)\right] \\
&= k_{1}\left[p\left(u, z_{2 n}\right)+p\left(v, w_{2 n}\right)\right]+k_{2}[p(u, F(s, t))+p(v, F(t, s))] \\
&+k_{3}\left[p\left(z_{2 n}, z_{2 n+1}\right)+p\left(w_{2 n}, w_{2 n+1}\right)\right]+k_{4}\left[p\left(u, z_{2 n+1}\right)+p\left(v, w_{2 n+1}\right)\right] \\
&+k_{5}\left[p\left(z_{2 n}, F(s, t)\right)+p\left(w_{2 n}, F(t, s)\right)\right] \\
& \leq \quad k_{1}\left[p\left(u, z_{2 n}\right)+p\left(v, w_{2 n}\right)\right]+k_{2}[p(u, F(s, t))+p(v, F(t, s))] \\
&+k_{3}\left[p\left(z_{2 n}, z_{2 n+1}\right)+p\left(w_{2 n}, w_{2 n+1}\right)\right]+k_{4}\left[p\left(u, z_{2 n+1}\right)+p\left(v, w_{2 n+1}\right)\right] \\
&+k_{5}\left[p\left(z_{2 n}, z_{2 n+1}\right)+p\left(z_{2 n+1}, F(s, t)\right)-p\left(z_{2 n+1}, z_{2 n+1}\right)\right. \\
&\left.+p\left(w_{2 n}, w_{2 n+1}\right)+p\left(w_{2 n+1}, F(t, s)\right)-p\left(w_{2 n+1}, w_{2 n+1}\right)\right] \\
& \leq \quad k_{1}\left[p\left(u, z_{2 n}\right)+p\left(v, w_{2 n}\right)\right]+k_{2}[p(u, F(s, t))+p(v, F(t, s))] \\
&+k_{3}\left[p\left(z_{2 n}, z_{2 n+1}\right)+p\left(w_{2 n}, w_{2 n+1}\right)\right]+k_{4}\left[p\left(u, z_{2 n+1}\right)+p\left(v, w_{2 n+1}\right)\right] \\
&+k_{5}\left[p\left(z_{2 n}, z_{2 n+1}\right)+p\left(z_{2 n+1}, F(s, t)\right)+p\left(w_{2 n}, w_{2 n+1}\right)+p\left(w_{2 n+1}, F(t, s)\right)\right] .
\end{aligned}
$$

Letting $n \rightarrow \infty$ in the above inequality and using (2.9)-(2.14), we arrive at

$$
p(F(s, t), u)+p(F(t, s), v) \leq\left(k_{2}+k_{5}\right)[p(F(s, t), u)+p(F(t, s), v)] .
$$

By (2.1), we have that $k_{2}+k_{5}<1$. Hence, it follows from (2.15) that $p(F(s, t), u)=p(F(t, s), v)=0$. By Lemma 1.8, we get $F(s, t)=u=f s$ and $F(t, s)=v=f t$. Hence, $(s, t)$ is a coincidence point of 
mappings $F$ and $f$. Since $(F, f)$ is $w$-compatible, we have $f u=f(F(s, t))=F(f s, f t)=F(u, v)$ and $f v=f(F(t, s))=F(f t, f s)=F(v, u)$. Suppose that $f u \neq u$ or $f v \neq v$. Hence

$$
d_{p}\left(f u, z_{2 n}\right)=2 p\left(f u, z_{2 n}\right)-p(f u, f u)-p\left(z_{2 n}, z_{2 n}\right) .
$$

Letting $n \rightarrow \infty$, we get

$$
d_{p}(f u, u)=2 \lim _{n \rightarrow \infty} p\left(f u, z_{2 n}\right)-p(f u, f u)
$$

which implies that

$$
2 p(f u, u)-p(f u, f u)-p(u, u)=d_{p}(f u, u)=2 \lim _{n \rightarrow \infty} p\left(f u, z_{2 n}\right)-p(f u, f u) .
$$

By using (2.12), we obtain

$$
p(f u, u)=\lim _{n \rightarrow \infty} p\left(f u, z_{2 n}\right)
$$

Similarly, we have

$$
p(f v, v)=\lim _{n \rightarrow \infty} p\left(f v, w_{2 n}\right)
$$

Thus

$$
\lim _{n \rightarrow \infty}\left[p\left(f u, z_{2 n}\right)+p\left(f v, w_{2 n}\right)\right]=p(f u, u)+p(f v, v)>0 .
$$

From (2.2), (2.3), $\left(p_{2}\right)$ and $\left(p_{4}\right)$, we get

$$
\begin{aligned}
& p(f u, u)+p(f v, v) \\
& \leq \quad p\left(f u, z_{2 n+1}\right)+p\left(z_{2 n+1}, u\right)-p\left(z_{2 n+1}, z_{2 n+1}\right) \\
&+p\left(f v, w_{2 n+1}\right)+p\left(w_{2 n+1}, v\right)-p\left(w_{2 n+1}, w_{2 n+1}\right) \\
& \leq p\left(f u, z_{2 n+1}\right)+p\left(z_{2 n+1}, u\right)+p\left(f v, w_{2 n+1}\right)+p\left(w_{2 n+1}, v\right) \\
&= p\left(F(u, v), G\left(x_{2 n+1}, y_{2 n+1}\right)\right)+p\left(F(v, u), G\left(y_{2 n+1}, x_{2 n+1}\right)\right)+p\left(z_{2 n+1}, u\right)+p\left(w_{2 n+1}, v\right) \\
& \leq \quad k_{1}\left[p\left(f u, g x_{2 n+1}\right)+p\left(f v, g y_{2 n+1}\right)\right]+k_{2}[p(f u, F(u, v))+p(f v, F(v, u))] \\
&+k_{3}\left[p\left(g x_{2 n+1}, G\left(x_{2 n+1}, y_{2 n+1}\right)\right)+p\left(g y_{2 n+1}, G\left(y_{2 n+1}, x_{2 n+1}\right)\right)\right] \\
&+k_{4}\left[p\left(f u, G\left(x_{2 n+1}, y_{2 n+1}\right)\right)+p\left(f v, G\left(y_{2 n+1}, x_{2 n+1}\right)\right)\right] \\
&+k_{5}\left[p\left(g x_{2 n+1}, F(u, v)\right)+p\left(g y_{2 n+1}, F(v, u)\right)\right]+p\left(z_{2 n+1}, u\right)+p\left(w_{2 n+1}, v\right) \\
&=\left.k_{1}\left[p\left(f u, z_{2 n}\right)+p\left(f v, w_{2 n}\right)\right]+k_{2}[p(f u, f u)+p(f v, f v))\right] \\
&+k_{3}\left[p\left(z_{2 n}, z_{2 n+1}\right)+p\left(w_{2 n}, w_{2 n+1}\right)\right]+k_{4}\left[p\left(f u, z_{2 n+1}\right)+p\left(f v, w_{2 n+1}\right)\right] \\
&\left.+k_{5}\left[p\left(z_{2 n}, f u\right)+p\left(w_{2 n}, f v\right)\right)\right]+p\left(z_{2 n+1}, u\right)+p\left(w_{2 n+1}, v\right) \\
& \leq \quad\left.k_{1}\left[p\left(f u, z_{2 n}\right)+p\left(f v, w_{2 n}\right)\right]+k_{2}[p(f u, u)+p(f v, v))\right] \\
&+k_{3}\left[p\left(z_{2 n}, z_{2 n+1}\right)+p\left(w_{2 n}, w_{2 n+1}\right)\right]+k_{4}\left[p\left(f u, z_{2 n+1}\right)+p\left(f v, w_{2 n+1}\right)\right] \\
&+k_{5}\left[p\left(f u, z_{2 n}\right)+p\left(f v, w_{2 n}\right)\right]+p\left(z_{2 n+1}, u\right)+p\left(w_{2 n+1}, v\right) .
\end{aligned}
$$

Letting $n \rightarrow \infty$ in the above inequality, using (2.9)-(2.12) and (2.16)-(2.18), we obtain

$$
\left.p(f u, u)+p(f v, v) \leq\left(k_{1}+k_{2}+k_{4}+k_{5}\right)\right)[p(f u, u)+p(f v, v)]<p(f u, u)+p(f v, v) .
$$

It is a contradiction. Hence $f u=u$ and $f v=v$. Thus

$$
F(u, v)=f u=u \text { and } F(v, u)=f v=v .
$$


Since $F(X \times X) \subset g X$, there exist $a, b \in X$ such that $u=F(u, v)=g a$ and $v=F(v, u)=g b$. Hence, it follows from (2.2) that

$$
\begin{aligned}
& p(u, G(a, b))+p(v, G(b, a)) \\
&=\quad p(F(u, v), G(a, b))+p(F(v, u), G(b, a)) \\
& \leq \quad k_{1}[p(f u, g a)+p(f v, g b)]+k_{2}[p(f u, F(u, v))+p(f v, F(v, u))] \\
&+k_{3}[p(g a, G(a, b))+p(g b, G(b, a))]+k_{4}[p(f u, G(a, b))+p(f v, G(b, a))] \\
&+k_{5}[p(g a, F(u, v))+p(g b, F(v, u))] \\
&=\left.\left.k_{1}[p(u, u)+p(v, v)]+k_{2}[p(u, u))+p(v, v)\right)\right] \\
&+k_{3}[p(u, G(a, b))+p(v, G(b, a))]+k_{4}[p(u, G(a, b))+p(v, G(b, a))] \\
&\left.\left.+k_{5}[p(u, u))+p(v, v)\right)\right] \\
&=\left(k_{3}+k_{4}\right)[p(u, G(a, b))+p(v, G(b, a))]
\end{aligned}
$$

which implies that $p(u, G(a, b))+p(v, G(b, a))=0$. So, $G(a, b)=u=g a$ and $G(b, a)=v=g b$. Since the pair $(G, g)$ is $w$-compatible, we have $g u=g(G(a, b))=G(g a, g b)=G(u, v)$ and $g v=g(G(b, a))=$ $G(g b, g a)=G(v, u)$. Suppose $g u \neq u$ or $g v \neq v$. It follows from (2.2) that

$$
\begin{aligned}
p(u, g u) & +p(v, g v) \\
= & p(F(u, v), G(u, v))+p(F(v, u), G(v, u)) \\
\leq \quad & k_{1}[p(f u, g u)+p(f v, g v)]+k_{2}[p(f u, F(u, v))+p(f v, F(v, u))] \\
& +k_{3}[p(g u, G(u, v))+p(g v, G(v, u))]+k_{4}[p(f u, G(u, v))+p(f v, G(v, u))] \\
& +k_{5}[p(g u, F(u, v))+p(g v, F(v, u))] \\
= & \left.\left.k_{1}[p(u, g u)+p(v, g v)]+k_{2}[p(u, u))+p(v, v)\right)\right] \\
& \left.\left.+k_{3}[p(g u, g u)+p(g v, g v)]+k_{4}[p(u, g u))+p(v, g v)\right)\right] \\
& \left.\left.+k_{5}[p(g u, u))+p(g v, v)\right)\right] \\
\leq \quad & \left.\left.k_{1}[p(u, g u)+p(v, g v)]+k_{2}[p(u, u))+p(v, v)\right)\right] \\
& \left.\left.+k_{3}[p(u, g u)+p(v, g v)]+k_{4}[p(u, g u))+p(v, g v)\right)\right] \\
& \left.\left.+k_{5}[p(u, g u))+p(v, g v)\right)\right] \\
= & \left(k_{1}+k_{3}+k_{4}+k_{5}\right)[p(u, g u)+p(v, g v)] \\
< & p(u, g u)+p(v, g v),
\end{aligned}
$$

which is a contradiction. It follows that $g u=u$ and $g v=v$. Hence

$$
G(u, v)=g u=u \text { and } G(v, u)=g v=v .
$$

From (2.19) and (2.20), it follows that $(u, v)$ is a common coupled fixed point of $F, G, f$ and $g$. 
Next, we show that $(u, v)$ is unique common coupled fixed point of $F, G, f$ and $g$. Suppose that $\left(u^{*}, v^{*}\right)$ be another common coupled fixed point of $F, G, f$ and $g$. Using (2.2) and ( $\left.p_{2}\right)$, we obtain

$$
\begin{aligned}
& p\left(u, u^{*}\right)+p\left(v, v^{*}\right) \\
&=\quad p\left(F(u, v), G\left(u^{*}, v^{*}\right)\right)+p\left(F(v, u), G\left(v^{*}, u^{*}\right)\right) \\
& \leq \quad k_{1}\left[p\left(f u, g u^{*}\right)+p\left(f v, g v^{*}\right)\right]+k_{2}[p(f u, F(u, v))+p(f v, F(v, u))] \\
&+k_{3}\left[p\left(g u^{*}, G\left(u^{*}, v^{*}\right)\right)+p\left(g v^{*}, G\left(v^{*}, u^{*}\right)\right)\right]+k_{4}\left[p\left(f u, G\left(u^{*}, v^{*}\right)\right)+p\left(f v, G\left(v^{*}, u^{*}\right)\right)\right] \\
&+k_{5}\left[p\left(g u^{*}, F(u, v)\right)+p\left(g v^{*}, F(v, u)\right)\right] \\
&= k_{1}\left[p\left(u, u^{*}\right)+p\left(v, v^{*}\right)\right]+k_{2}[p(u, u)+p(v, v)]+k_{3}\left[p\left(u^{*}, u^{*}\right)+p\left(v^{*}, v^{*}\right)\right] \\
&+k_{4}\left[p\left(u, u^{*}\right)+p\left(v, v^{*}\right)\right]+k_{5}\left[p\left(u^{*}, u\right)+p\left(v^{*}, v\right)\right] \\
& \leq \quad k_{1}\left[p\left(u, u^{*}\right)+p\left(v, v^{*}\right)\right]+k_{2}\left[p\left(u, u^{*}\right)+p\left(v, v^{*}\right)\right]+k_{3}\left[p\left(u, u^{*}\right)+p\left(v, v^{*}\right)\right] \\
&+k_{4}\left[p\left(u, u^{*}\right)+p\left(v, v^{*}\right)\right]+k_{5}\left[p\left(u, u^{*}\right)+p\left(v, v^{*}\right)\right] \\
&=\left(k_{1}+k_{2}+k_{3}+k_{4}+k_{5}\right)\left[p\left(u, u^{*}\right)+p\left(v, v^{*}\right)\right] .
\end{aligned}
$$

From (2.2) and (2.21), we have $p\left(u, u^{*}\right)=p\left(v, v^{*}\right)=0$. By Lemma 1.8, we get $u^{*}=u$ and $v^{*}=v$, which implies that the uniqueness of the common coupled fixed point of $F, G, f$ and $g$.

Next, we show that $u=v$. In fact, we find from (2.2) and (2.12) that

$$
\begin{aligned}
p(u, v) & +p(v, u) \\
= & p(F(u, v), G(v, u))+p(F(v, u), G(u, v)) \\
\leq \quad & k_{1}[p(f u, g v)+p(f v, g u)]+k_{2}[p(f u, F(u, v))+p(f v, F(v, u))] \\
& +k_{3}[p(g v, G(v, u))+p(g u, G(u, v))]+k_{4}[p(f u, G(v, u))+p(f v, G(u, v))] \\
& +k_{5}[p(g v, F(u, v))+p(g u, F(v, u))] \\
= & k_{1}[p(u, v)+p(v, u)]+k_{2}[p(u, u)+p(v, v)]+k_{3}[p(v, v)+p(u, u)] \\
& +k_{4}[p(u, v)+p(v, u)]+k_{5}[p(v, u)+p(u, v)] \\
= & \left(k_{1}+k_{4}+k_{5}\right)[p(u, v)+p(v, u)] .
\end{aligned}
$$

From (2.1), we have $p(u, v)=p(v, u)=0$. By Lemma 1.8, we get that $u=v$. Thus $u=f u=F(u, u)=$ $G(u, u)=g u$, that is, the common coupled fixed point of $F, G, f$ and $g$ has the form $(u, u)$. If $g(X)$ is a complete subspace of $X$, we obtain the desired conclusion immediately.

Corollary 2.2. Let $(X, p)$ be a partial metric space. Let $F, G: X \times X \rightarrow X, f, g: X \rightarrow X$ be four mappings. Suppose that there exist $k \in[0,1)$ such that

$$
p(F(x, y), G(u, v))+p(F(y, x), G(v, u)) \leq k[p(f x, g u)+p(f y, g v)]
$$

for all $x, y, u, v \in X$. Also, suppose the following hypotheses:

(i) $F(X \times X) \subset g(X)$ and $G(X \times X) \subset f(X)$,

(ii) either $f(X)$ or $g(X)$ is a complete subspace of $X$,

(iii) $(F, f)$ and $(G, g)$ are w-compatible.

Then $F, G, f$ and $g$ have a unique common coupled fixed point in $X \times X$. Moreover, the common coupled fixed point of $F, G, f$ and $g$ have the form $(u, u)$. 
Corollary 2.3. Let $(X, p)$ be a partial metric space. Let $F, G: X \times X \rightarrow X, g: X \rightarrow X$ be three mappings. Suppose that there exist $k \in[0,1)$ such that

$$
p(F(x, y), G(u, v))+p(F(y, x), G(v, u)) \leq k[p(g u, F(x, y))+p(g v, F(y, x))]
$$

for all $x, y, u, v \in X$. Also, suppose the following hypotheses:

(i) $F(X \times X) \subset g(X)$ and $G(X \times X) \subset g(X)$,

(ii) $g(X)$ is a complete subspace of $X$,

(iii) $(F, g)$ and $(G, g)$ are w-compatible.

Then $F, G$ and $g$ have a unique common coupled fixed point in $X \times X$. Moreover, the common coupled fixed point of $F, G$ and $g$ have the form $(u, u)$.

Theorem 2.4. Let $(X, p)$ be a partial metric space. Let $F, G: X \times X \rightarrow X, f, g: X \rightarrow X$ be four mappings. Suppose that there exist $a_{i} \in[0,1)(i=1,2,3, \cdots, 10)$ with

$$
a_{1}+a_{2}+a_{3}+a_{4}+a_{5}+a_{6}+2\left(a_{7}+a_{8}+a_{9}+a_{10}\right)<1
$$

such that

$$
\begin{aligned}
& p(F(x, y), G(u, v)) \\
& \leq \quad a_{1} p(f x, g u)+a_{2} p(f y, g v)+a_{3} p(f x, F(x, y))+a_{4} p(f y, F(y, x)) \\
& \quad+a_{5} p(g u, G(u, v))+a_{6} p(g v, G(v, u))+a_{7} p(f x, G(u, v))+a_{8} p(f y, G(v, u)) \\
& \quad+a_{9} p(g u, F(x, y))+a_{10} p(g v, F(y, x))
\end{aligned}
$$

for all $x, y, u, v \in X$. Also, suppose the following hypotheses:

(i) $F(X \times X) \subset g(X)$ and $G(X \times X) \subset f(X)$,

(ii) either $f(X)$ or $g(X)$ is a complete subspace of $X$,

(iii) $(F, f)$ and $(G, g)$ are w-compatible.

Then $F, G, f$ and $g$ have a unique common coupled fixed point in $X \times X$. Moreover, the common coupled fixed point of $F, G, f$ and $g$ have the form $(u, u)$.

Proof. Fix $x, y, u, v \in X$. It follows from (2.25) that

$$
\begin{aligned}
p(F(x, y), G(u, v)) & \\
\leq & a_{1} p(f x, g u)+a_{2} p(f y, g v)+a_{3} p(f x, F(x, y))+a_{4} p(f y, F(y, x)) \\
& +a_{5} p(g u, G(u, v))+a_{6} p(g v, G(v, u))+a_{7} p(f x, G(u, v))+a_{8} p(f y, G(v, u)) \\
& +a_{9} p(g u, F(x, y))+a_{10} p(g v, F(y, x))
\end{aligned}
$$

and

$$
\begin{aligned}
& p(F(y, x), G(v, u)) \\
& \leq \quad a_{1} p(f y, g v)+a_{2} p(f x, g u)+a_{3} p(f y, F(y, x))+a_{4} p(f x, F(x, y)) \\
&+a_{5} p(g v, G(v, u))+a_{6} p(g u, G(u, v))+a_{7} p(f y, G(v, u))+a_{8} p(f x, G(u, c)) \\
&+a_{9} p(g v, F(y, x))+a_{10} p(g u, F(x, y)) .
\end{aligned}
$$


From (2.26) and (2.27), we get

$$
\begin{aligned}
& p(F(x, y), G(u, v))+p(F(y, x), G(v, u)) \\
& \leq \quad\left(a_{1}+a_{2}\right)[p(f x, g u)+p(f y, g v)]+\left(a_{3}+a_{4}\right)[p(f x, F(x, y))+p(f y, F(y, x))] \\
&+\left(a_{5}+a_{6}\right)[p(g u, G(u, v))+p(g v, G(v, u))]+\left(a_{7}+a_{8}\right)[p(f x, G(u, v))+p(f y, G(v, u))] \\
&+\left(a_{9}+a_{10}\right)[p(g u, F(x, y))+p(g v, F(y, x))] .
\end{aligned}
$$

Therefore, the result follows from Theorem 2.4 immediately.

Remark 2.5. Theorem 2.4 improves and extends Theorem 1.7 from one mapping to two hybrid pairs of mappings, and the contractive condition (1.2) is replaced by the new contractive condition defined by (2.25).

Remark 2.6. Corollary 2.3 of Aydi and Abbas [14] is a particular case of Theorem 2.4 by taking $a_{4}=$ $a_{6}=a_{8}=a_{10}=0, F=G$ and $f=g=I$, the identity on $X$.

Corollary 2.7. Let $(X, p)$ be a partial metric space. Let $F, G: X \times X \rightarrow X, f: X \rightarrow X$ be three mappings. Suppose that there exist $k, l \in[0,1)$ with $k+l<1$ such that

$$
p(F(x, y), G(u, v)) \leq k p(f x, g u)+l p(f y, g v)
$$

for all $x, y, u, v \in X$. Also, suppose the following hypotheses:

(i) $F(X \times X) \subset f(X)$ and $G(X \times X) \subset f(X)$,

(ii) $f(X)$ is a complete subspace of $X$,

(iii) $(F, f)$ and $(G, f)$ are w-compatible.

Then $F, G$ and $f$ have a unique common coupled fixed point in $X \times X$. Moreover, the common coupled fixed point of $F, G$ and $f$ have the form $(u, u)$.

Remark 2.8. Corollary 2.7 generalizes and extends the corresponding results in Aydi [13, Theorem 2.1] from one mapping to two hybrid pairs of mappings. In fact, if $F=G$ and $f=g=I$, Corollary 2.7 is reduced to Theorem 2.1 of Aydi [13].

Corollary 2.9. Let $(X, p)$ be a partial metric space. Let $F, G: X \times X \rightarrow X, f, g: X \rightarrow X$ be four mappings. Suppose that there exist $k, l \in[0,1)$ with $k+l<1$ such that

$$
p(F(x, y), G(u, v)) \leq k p(f x, F(x, y))+\operatorname{lp}(g u, G(u, v))
$$

for all $x, y, u, v \in X$. Also, suppose the following hypotheses:

(i) $F(X \times X) \subset g(X)$ and $G(X \times X) \subset f(X)$,

(ii) $f(X)$ or $g(X)$ is a complete subspace of $X$.

(iii) $(F, f)$ and $(G, g)$ are w-compatible.

Then $F, G, f$ and $g$ have a unique common coupled fixed point in $X \times X$. Moreover, the common coupled fixed point of $F, G, f$ and $g$ have the form $(u, u)$.

Remark 2.10. (1) If $F=G$ and $f=g=I$ in Corollary 2.9, then Corollary 2.9 is reduced to Theorem 2.4 of Aydi [13].

(2) If we take $F=G, f=g=I$ and $k=l$, then Corollary 2.9 is reduced to Corollary 2.6 of Aydi [13]. 
Corollary 2.11. Let $(X, p)$ be a partial metric space. Let $F, G: X \times X \rightarrow X, f, g: X \rightarrow X$ be four mappings. Suppose that there exist $k, l \in[0,1)$ with $k+l<\frac{1}{2}$ such that

$$
p(F(x, y), G(u, v)) \leq k p(f x, G(u, v))+\operatorname{lp}(g u, F(x, y))
$$

for all $x, y, u, v \in X$. Also, suppose the following hypotheses:

(i) $F(X \times X) \subset g(X)$ and $G(X \times X) \subset f(X)$,

(ii) $f(X)$ or $g(X)$ is a complete subspace of $X$,

(iii) $(F, f)$ and $(G, g)$ are w-compatible.

Then $F, G, f$ and $g$ have a unique common coupled fixed point in $X \times X$. Moreover, the common coupled fixed point of $F, G, f$ and $g$ have the form $(u, u)$.

Remark 2.12. (1) If $F=G$ and $f=g=I$ in Corollary 2.11, Corollary 2.11 is reduced to Theorem 2.5 of Aydi [13].

(2) If $F=G, f=g=I$ and $k=l$ in Corollary 2.11, Corollary 2.11 is reduced to Corollary 2.7 of Aydi [13].

Corollary 2.13. Let $(X, p)$ be a partial metric space. Let $F, G: X \times X \rightarrow X, f, g: X \rightarrow X$ be four mappings. Suppose that there exist $k, l \in[0,1)$ with $k+l<\frac{1}{2}$ such that

$$
p(F(x, y), G(u, v)) \leq k p(f y, G(v, u))+l p(g v, F(y, x))
$$

holds for all $x, y, u, v \in X$. Also, suppose the following hypotheses:

(i) $F(X \times X) \subset g(X)$ and $G(X \times X) \subset f(X)$,

(ii) either $f(X)$ or $g(X)$ is a complete subspace of $X$,

(iii) $(F, f)$ and $(G, g)$ are w-compatible.

Then $F, G, f$ and $g$ have a unique common coupled fixed point in $X \times X$. Moreover, the common coupled fixed point of $F, G, f$ and $g$ have the form $(u, u)$.

Let $f=g=I$ in Theorem 2.4 and Corollaries 2.7-2.13, we have the following results.

Corollary 2.14. Let $(X, p)$ be a complete partial metric space. Let $F, G: X \times X \rightarrow X$ be two mappings. Suppose that there exist $a_{i} \in[0,1)(i=1,2,3, \cdots, 10)$ with

$$
\begin{aligned}
& a_{1}+a_{2}+a_{3}+a_{4}+a_{5}+a_{6}+2\left(a_{7}+a_{8}+a_{9}+a_{10}\right)<1 \text { such that } \\
& \qquad \begin{aligned}
& p(F(x, y), G(u, v)) \\
& \leq a_{1} p(x, u)+a_{2} p(y, v)+a_{3} p(x, F(x, y))+a_{4} p(y, F(y, x)) \\
&+a_{5} p(u, G(u, v))+a_{6} p(v, G(v, u))+a_{7} p(x, G(u, v))+a_{8} p(y, G(v, u)) \\
&+a_{9} p(u, F(x, y))+a_{10} p(v, F(y, x))
\end{aligned}
\end{aligned}
$$

for all $x, y, u, v \in X$. Then $F$ and $G$ have a unique common coupled fixed point in $X \times X$. Moreover, the common coupled fixed point of $F$ and $G$ have the form $(u, u)$.

Remark 2.15. Corollary 2.14 improves and extends the corresponding results in Aydi [13, Theorem 2.1] from one self-mappings to two self-mappings, and the contractive condition is replaced by the new contractive condition defined by (2.33). 
Corollary 2.16. Let $(X, p)$ be a complete partial metric space. Let $F, G: X \times X \rightarrow X$ be two mappings. Suppose that there exist $k, l \in[0,1)$ with $k+l<1$ such that

$$
p(F(x, y), G(u, v)) \leq k p(x, u)+l p(y, v)
$$

for all $x, y, u, v \in X$. Then $F$ and $G$ have a unique common coupled fixed point in $X \times X$. Moreover, the common coupled fixed point of $F$ and $G$ have the form $(u, u)$.

Remark 2.17. (1) If $F=G$ in Corollary 2.16, then Corollary 2.16 is reduced to Theorem 2.1 of Aydi [13].

(1) If $F=G$ and $k=l$ in Corollary 2.16, then Corollary 2.16 is reduced to Corollary 2.2 of Aydi [13].

Corollary 2.18. Let $(X, p)$ be a complete partial metric space. Let $F, G: X \times X \rightarrow X$ be two mappings. Suppose that there exist $k, l \in[0,1)$ with $k+l<1$ such that

$$
p(F(x, y), G(u, v)) \leq k p(x, F(x, y))+\operatorname{lp}(u, G(u, v))
$$

for all $x, y, u, v \in X$. Then $F$ and $G$ have a unique common coupled fixed point in $X \times X$. Moreover, the common coupled fixed point of $F$ and $G$ have the form $(u, u)$.

Remark 2.19. (1) If $F=G$ in Corollary 2.18, then Corollary 2.18 is reduced to Theorem 2.4 of Aydi [13].

(2) If $F=G$ and $k=l$ in Corollary 2.18, then Corollary 2.18 is reduced to Corollary 2.6 of Aydi [13].

Corollary 2.20. Let $(X, p)$ be a complete partial metric space. Let $F, G: X \times X \rightarrow X$ be two mappings. Suppose that there exist $k, l \in[0,1)$ with $k+l<\frac{1}{2}$ such that

$$
p(F(x, y), G(u, v)) \leq k p(x, G(u, v))+\operatorname{lp}(u, F(x, y))
$$

for all $x, y, u, v \in X$. Then $F$ and $G$ have a unique common coupled fixed point in $X \times X$. Moreover, the common coupled fixed point of $F$ and $G$ have the form $(u, u)$.

Remark 2.21. (1) If $F=G$ in Corollary 2.20, then Corollary 2.20 is reduced to Theorem 2.5 of Aydi [13].

(1) If $F=G$ and $k=l$ in Corollary 2.20, then Corollary 2.20 is reduced to Corollary 2.7 of Aydi [13].

Now, we give some examples to support our main results.

Example 2.22. Let $X=[0,1]$ be a partial metric space with $p(x, y)=\max \{x, y\}$, and let the mappings $F, G: X \times X \rightarrow X$ and $f, g: X \times X \rightarrow X$ be defined by

for all $x, y \in X$. Then

$$
F(x, y)=\frac{x^{2}+y^{2}}{6}, G(x, y)=\frac{x+y}{12}, f x=x^{2} \text { and } g x=\frac{x}{2}
$$

(i) $F(X \times X) \subset g(X)$ and $G(X \times X) \subset f(X)$,

(ii) either $f(X)$ is a complete subspace of $X$,

(iii) $(F, f)$ and $(G, g)$ are $w$-compatible,

(iv) for any $x, y, u, v \in X$, we have

$$
p(F(x, y), G(u, v))+p(F(y, x), G(v, u)) \leq \frac{1}{3}[p(f x, g u)+p(f y, g v)] .
$$


Proof. (i), (ii) and (iii) are obvious. Next we show that (iv). In fact, for $x, y, u, v \in X$, we have

$$
\begin{aligned}
p(F(x, y), G(u, v))+p(F(y, x), G(v, u)) \\
=2 \max \left\{\frac{x^{2}+y^{2}}{6}, \frac{u+v}{12}\right\} \\
\leq 2\left(\max \left\{\frac{x^{2}}{6}, \frac{u}{12}\right\}+\max \left\{\frac{y^{2}}{6}, \frac{v}{12}\right\}\right) \\
=\frac{1}{3}\left(\max \left\{x^{2}, \frac{u}{2}\right\}+\max \left\{y^{2}, \frac{v}{2}\right\}\right) \\
=\frac{1}{3}[p(f x, g u)+p(f y, g v)] .
\end{aligned}
$$

Thus, $F, G, f$ and $g$ satisfy all the hypotheses of Corollary 2.2. So, $F, G, f$ and $g$ have a unique common coupled fixed point. Here $(0,0)$ is the unique common coupled fixed point of $F, G, f$ and $g$.

Example 2.23. Let $X=R^{+}$be a partial metric space with $p(x, y)=\max \{x, y\}$. Let $F, G: X \times X \rightarrow X$ and $f, g: X \times X \rightarrow X$ be mapping defined by

$$
F(x, y)=\frac{2 x+3 y}{72}, G(x, y)=\frac{3 x+4 y}{72}, f x=\frac{x}{6} \text { and } g x=\frac{x}{2}, \quad \forall x, y \in X .
$$

We find that all the hypotheses of Corollary 2.7 are satisfied. Clearly, $F(X \times X) \subset g(X), G(X \times X) \subset$ $f(X), f(X)$ is a complete subspace of $X$, and the pairs $(F, f)$ and $(G, g)$ are $w$-compatible. For all $x, y, u, v \in X$, we have

$$
\begin{aligned}
p(F(x, y), G(u, v)) & =\max \left\{\frac{2 x+3 y}{72}, \frac{3 u+4 v}{72}\right\} \\
& =\max \left\{\frac{x}{36}+\frac{y}{24}, \frac{u}{24}+\frac{v}{18}\right\} \\
& \leq \max \left\{\frac{x}{36}, \frac{u}{24}\right\}+\max \left\{\frac{y}{24}, \frac{v}{18}\right\} \\
& \leq \max \left\{\frac{x}{36}, \frac{u}{12}\right\}+\max \left\{\frac{y}{24}, \frac{v}{8}\right\} \\
& =\frac{1}{6} \max \left\{\frac{x}{6}, \frac{u}{2}\right\}+\frac{1}{4} \max \left\{\frac{y}{6}, \frac{v}{2}\right\} \\
& =\frac{1}{6} p(f x, g u)+\frac{1}{4} p(f y, g v) .
\end{aligned}
$$

Thus, $F, G, f$ and $g$ satisfy all the hypotheses of Corollary 2.7. So, $F, G, f$ and $g$ have a unique common coupled fixed point. Here $(0,0)$ is the unique common coupled fixed point of $F, G, f$ and $g$.

Example 2.24. Let $X=\{0,1,2\}$ be endowed with the partial metric $p$ given by $p(x, y)=\max \{x, y\}$ for all $x, y \in X$. It is clear that $(X, p)$ is a complete partial metric. Define the mappings $F, G: X \times X \rightarrow X$ and $f, g: X \rightarrow X$ by

TABLE 1. The definition of maps $f$ and $g$ on $X$

\begin{tabular}{ccc}
\hline$x$ & $f(x)$ & $g(x)$ \\
\hline 0 & 0 & 0 \\
1 & 2 & 2 \\
2 & 1 & 2 \\
\hline
\end{tabular}


and

TABLE 2. The definition of maps $F$ and $G$ on $X \times X$

\begin{tabular}{ccc}
\hline$(x, y)$ & $F(x, y)$ & $G(x, y)$ \\
\hline$(0,0)$ & 0 & 0 \\
$(0,1)$ & 0 & 0 \\
$(0,2)$ & 0 & 0 \\
$(1,0)$ & 0 & 0 \\
$(1,1)$ & 0 & 0 \\
$(1,2)$ & 0 & 0 \\
$(2,0)$ & 0 & 1 \\
$(2,1)$ & 0 & 1 \\
$(2,2)$ & 0 & 1 \\
\hline
\end{tabular}

Clearly, $f(X)=X$ is complete, $F(X \times X) \subseteq g(X)$ and $G(X \times X) \subseteq f(X)$. It is easy to show that $(F, f)$ and $(G, g)$ are $w$-weakly compatible. To check contractive condition (2.30) for all $x, y \in X, k=\frac{1}{3}$ and $l=\frac{1}{2}$, we consider the following cases:

Case $(I)(x, y) \in X \times X$ and $(u, v) \in\{(0,0),(0,1),(0,2),(1,0),(1,1),(1,2)\}$. Then $p(F(x, y), G(u, v))=$ $p(0,0)=0$. Hence $(2.30)$ is satisfied.

Case (II) $(x, y) \in X \times X$ and $(u, v) \in\{(2,0),(2,1),(2,2)\}$. Then

$$
\begin{aligned}
k p(f x, F(x, y))+l p(g u, G(u, v)) & \geq \operatorname{lp}(g u, G(u, v)) \\
& =\operatorname{lp}(2,1)=2 l=1 .
\end{aligned}
$$

Thus

$$
p(F(x, y), G(u, v))=p(0,1)=1 \leq k p(f x, F(x, y))+l p(g u, G(u, v)) .
$$

Hence, all of the conditions of Corollary 2.9 are satisfied. Moreover, $(0,0)$ is the unique common coupled fixed point of $F, G, f$ and $g$.

\section{Acknowledgements}

The first author was supported by the National Natural Science Foundation of China (Grant No. 11071169), and the Natural Science Foundation of Zhejiang Province (Grant No. Y6110287)

\section{REFERENCES}

[1] S. G. Matthews, Partial Metric Topology, Proc. 8th Summer of Conference on General Topology and Applications, Ann. New York Acad. Sci. 728 (1994), 183-197.

[2] Th. Abdeljawad, E. Karapinar, K. Taş, Existence and uniqueness of a common fixed point on partial metric spaces, Appl. Math. Lett. 24 (2011), 1900-1904.

[3] Th. Abdeljawad, Fixed points and generalized weakly contractive mappings in partial metric spaces, Math. Comput. Modelling, 54 (2011), 2923-2927.

[4] I. Altun, A. Erduran, Fixed point theorems for monotone mappings on partial metric spaces, Fixed Point Theory Appl. 2011 (2011), Article ID 508730.

[5] C. Chen, C. Zhu, Fixed point theorems for weakly $C$-contractive mappings in partial metric spaces, Fixed Point Theory Appl. 2013 (2013), Article ID 107. 
[6] D. Ilić, V. Pavlović, V. Rakočević, Fixed points of mappings with a contractive iterate at a point in partial metric spaces, Fixed Point Theory Appl. 2013 (2013), Article ID 335.

[7] S. Oltra, O. Valero, Banach's fixed point theorem for partial metric spaces, Rend. Ist. Mat. Univ. Trieste, 36 (2004), 17-26.

[8] D. Paesano, P. Vetro, Suzuki's type characterizations of completeness for partial metric spaces and fixed points for partially ordered metric spaces, Topology Appl. 159 (2012), 911-920.

[9] M. Nazam, I. Beg, M. Arshad, Common fixed points of weakly increasing F-contractions on ordered partial metric spaces, Commun. Optim. Theory 2019 (2019), Article ID 3.

[10] R. H. Haghi, Sh. Rezapour, N. Shahzad, Be careful on partial metric fixed point results, Topology Appl. 160 (2013), 450-454.

[11] T. G. Bhaskar, V. Lakshmikantham, Fixed point theorems in partially ordered metric spaces and applications, Nonlinear Anal. 65 (2006), 1379-1393.

[12] V. Lakshmikantham, Lj. Ćirić, Coupled fixed point theorems for nonlinear contractions in partially ordered metric spaces, Nonlinear Anal. 70 (2009), 4341-4349.

[13] H. Aydi, Some coupled fixed point results on partial metric spaces, Int. J. Math. Math. Sci. 2011 (2011), Article ID 647091.

[14] H. Aydi, M. Abbas, Tripled coincidence and fixed point results in partial metric spaces, Appl. General Topology 13 (2012), 193-206.

[15] F. Gu, Some common tripled fixed point results in two quasi-partial metric spaces, Fixed Point Theory Appl. 2014 (2014), Article ID 71.

[16] F. Gu, L. Wang, Some coupled fixed-point theorems in two quasi-partial metric spaces, Fixed Point Theory Appl. 2014 (2014), Article ID 19.

[17] F. Gu, Y. Yin, A new common coupled fixed point theorem in generalized metric space and applications to integral equations, Fixed Point Theory Appl. 2013 (2013), Article ID 266.

[18] M. Abbas, M. A. Khan, S. Radenovic, Common coupled fixed point theorem in cone metric space for $w$-compatible mappings, Appl. Math. Comput. 217 (2010), 195-202. 